\title{
Niveaux marins extrêmes et érosion du cordon dunaire de la plage du Vougot (commune de Guissény, Finistère). De la mesure à la réponse gestionnaire
}

\author{
Serge SUANEZ ${ }^{1}$, Bernard FICHAUT ${ }^{1}$, Luce GOUDEDRANCHE $^{2}$, \\ Lénaïg SPARFEL ${ }^{3}$
}

GÉOMER - UMR 6554 LETG - CNRS - Institut Universitaire Européen de la Mer - Place Nicolas Copernic, 29280 Plouzané serge.suanez@univ-brest.fr

${ }^{1}$ Maîtres de conférences à l'Université de Bretagne Occidentale

${ }^{2}$ Ingénieur - CETMEF (Brest)

${ }^{3}$ Doctorante au laboratoire GEOMER - LETG UMR 6554 CNRS

\section{Résumé}

Depuis plusieurs décennies la partie orientale du cordon dunaire de la plage du Vougot (commune de Guissény - Finistère) est en érosion. Cette dernière fait craindre aux riverains une éventuelle rupture du cordon avec pour conséquence une submersion marine de leur environnement. Aussi, à la demande de la commune une expertise scientifique a été réalisée afin d'analyser et de quantifier l'ampleur du phénomène érosif dans le but de proposer une solution techniques permettant de stabiliser le cordon dunaire.

\section{$\underline{\text { Abstract }}$}

The eastern section of the dune of Le Vougot beach (district of Guissény Finistère) has been facing erosion for the last decades. The enhabitants of this area fear that this erosion may break the dune and therefore induce marine submersion of their environment. Then, at the local authorities request, a scientific survey has been undertaken in order to analyse and quantify erosion phenomenon and to propose enginiery solution to stabilize the dune.

$\underline{\text { Mots clefs }}$ : cordon dunaire, érosion, expertise scientifique, solution technique.

\section{Problématique}

Situé sur la côte nord du Finistère, le cordon dunaire de la plage du Vougot (commune de Guissény) abrite un polder aménagé par l'homme au $19^{\text {ème }}$ siècle $^{[1]}$ afin d'augmenter la surface agricole ${ }^{[3]}$ (fig. 1). Il est aujourd'hui occupé par un étang (l'étang du Curnic) appartenant au Conservatoire du Littoral et jouit d'un statut de protection pour la diversité de sa faune et de sa flore. 
Une partie du polder où se trouvent des secteurs habités (camping, lotissements, fermes agricoles, etc.), reste vulnérable car son altitude est proche ou en dessous du niveau marin. Le cordon dunaire de la plage du Vougot joue donc le rôle de rempart naturel à une éventuelle submersion marine. Or, il s'avère que depuis quelques dizaines d'années, sa partie orientale est en érosion. Cette dernière est en grande partie attribuée à la construction en 1974 d'une jetée ${ }^{\text {[2] }}$ reliant l'îlot d'Enez Croaz-Hent à la partie orientale de la plage du Vougot afin de protéger la zone de mouillage du centre nautique du Curnic (fig. 1).

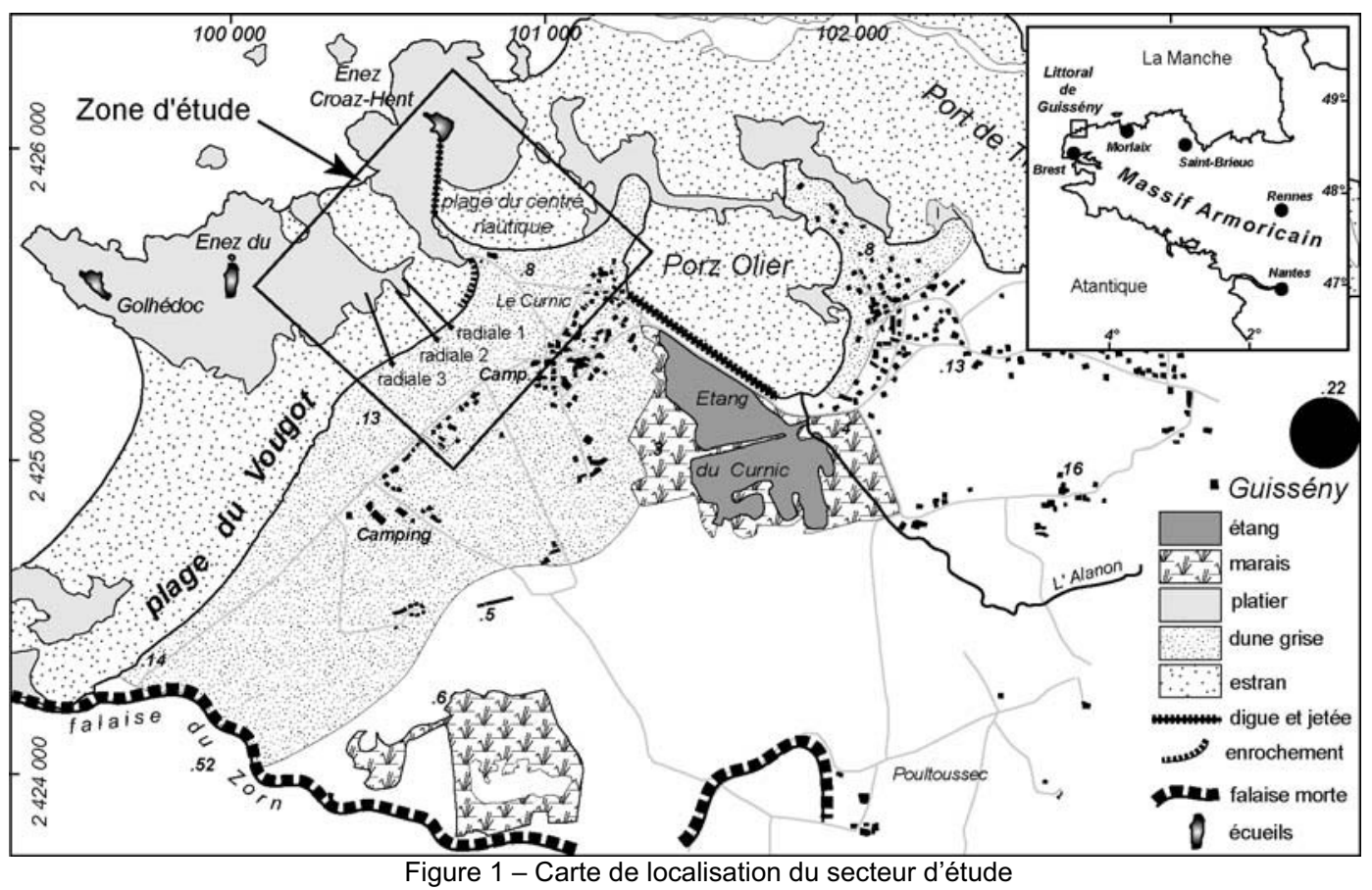

En effet, cet ouvrage fût mis en place sans qu'aucune expertise scientifique ne soit réalisée. Très rapidement le fonctionnement hydro-sédimentaire du secteur se modifia entraînant un recul du cordon dunaire. Pour lutter contre cette érosion, une solution de défense consistant à enrocher le pied de dune fût décidée par la commune en 1990. Mais là encore, sans qu'aucune étude technique ne soit réalisée, un enrochement fût posé sur $150 \mathrm{~m}$ de linéaire dans la section la plus orientale (fig. 1). Quinze ans après, les observations de terrain montrent que ce dernier ne fonctionne pas. L'érosion l'a depuis contourné et il est aujourd'hui, en partie, déconnecté du pied de dune (fig. 2).

Actuellement, le recul du cordon dunaire fait craindre aux habitants du polder une éventuelle rupture de ce dernier avec pour conséquence une submersion de leur environnement. Devant la pression des riverains, la commune a souhaité engager une expertise scientifique globale visant à analyser et à quantifier le phénomène d'érosion dunaire afin d'envisager une (ou des) solution(s) technique(s) le(s) plus appropriée(s) pour stopper le recul de la dune. 


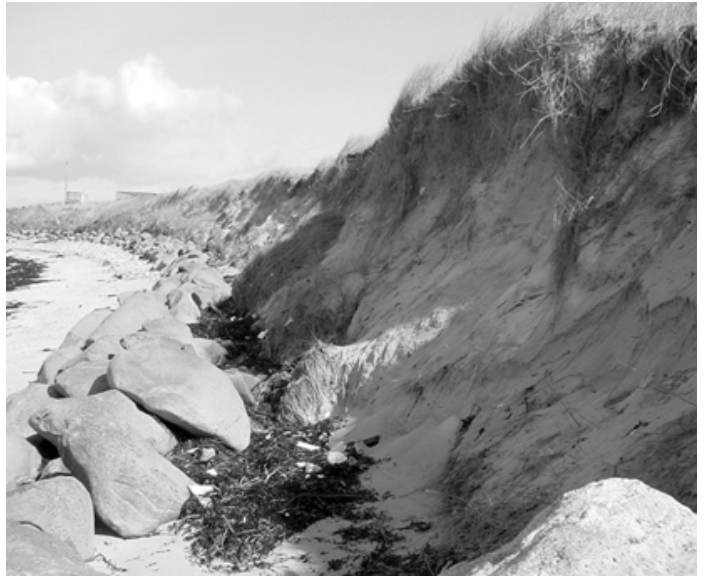

Figure 2 - Enrochement mis en place dans la section orientale du cordon dunaire aujourd'hui déconnecté du pied de dune par l'érosion marine qui le contourne.

\section{Méthodologie}

Ce travail d'expertise a été mené en collaboration avec le CETMEF pour la partie ingénierie. Dans un premier temps, une analyse diachronique par photointerprétation a été réalisée afin d'analyser et de quantifier la cinématique du front de dune depuis le début des années 1950. Dans un second temps, un suivi topomorphologique à haute fréquence (1 relevé par mois) a été entrepris à partir du mois de juin $2004^{[7]}$. Il repose sur des mesures de profils de plage/cordon dunaire au DGPS le long de trois radiales (fig. 1), avec une précision verticale de $\pm 2 \mathrm{~cm}$. Ce suivi s'est accompagné d'une analyse des conditions météo-marines enregistrées durant la période d'observation afin de préciser l'importance des niveaux marins extrêmes dans l'érosion du pied de dune. Enfin, l'ensemble de ce travail a débouché sur la proposition de solutions techniques.

\section{$\underline{\text { 3. Recul pluridécennal du cordon dunaire }}$}

Les travaux effectués par photogrammétrie montrent que le recul de la dune au niveau de la plage du Vougot a atteint environ $15 \mathrm{~m}$ entre 1952 et 2005, soit une vitesse de 0,3 m/an (fig. 3). Cette évolution s'est faite en deux temps, antérieurement et postérieurement à la construction de la jetée du Curnic en 1974 :

(i) entre 1952 et 1978, on observe une avancée du front de dune atteignant 5 à 8 $\mathrm{m}$. Dans le même temps, on note un recul très important (environ $30 \mathrm{~m}$ ) de la plage du centre nautique située à l'est. On peut en conclure que le matériel perdu par cette cellule venait alimenter celle de la plage du Vougot suivant un transit sédimentaire orienté d'est en ouest (fig. 3A).

(ii) à partir du début des années 1980, l'érosion du front de dune de la plage du Vougot commence. Elle atteint une vingtaine de mètres entre 1982 et 2005 (fig. 3B), la vitesse de recul est dans un premier temps de $1 \mathrm{~m} / \mathrm{an}$, à partir de 2000 elle passe à $1,5 \mathrm{~m} / \mathrm{an}$ (fig. 3C). Dans le même temps, on note un net ralentissement de l'érosion de la plage du centre nautique qui s'explique par le fait que la digue bloque désormais les sédiments à l'intérieur de cette cellule. 


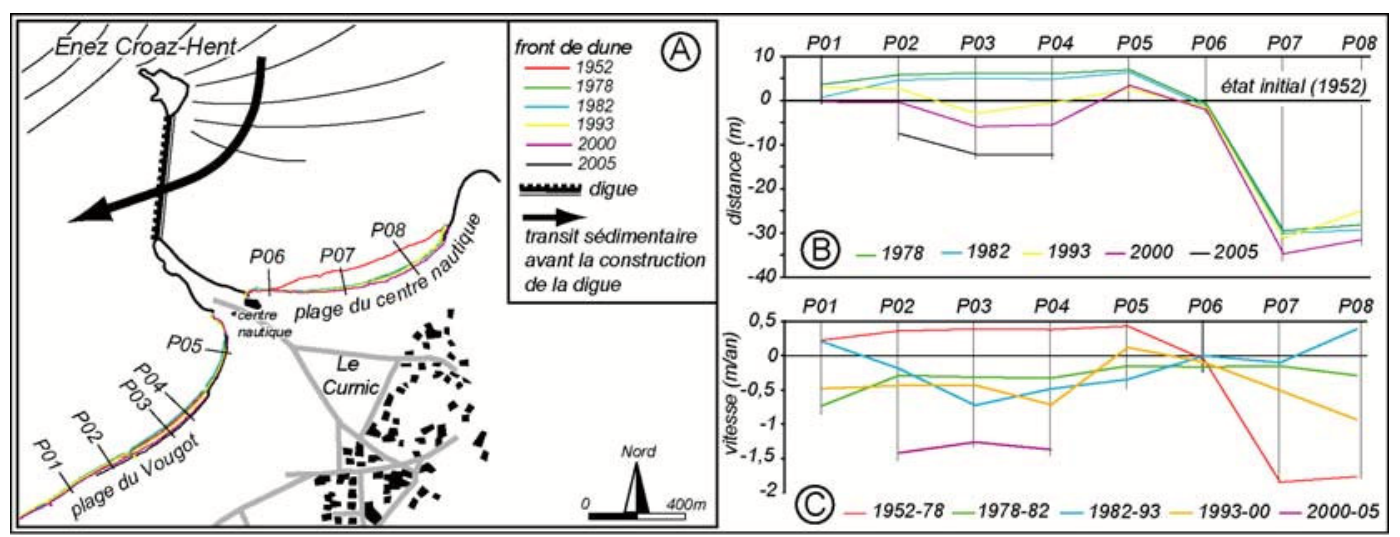

Figure 3 - Cinématique du front de dune de la plage du Vougot (P01 à P05) et du centre nautique (P06 à P08) entre 1952 et $2005-3 A$ et $3 B$. Vitesses de mobilité du front de dune $-3 C$.

\section{Suivi topo-morphologique du cordon dunaire}

Par souci de concision, nous ne présenterons que les résultats obtenus sur la radiale 1 qui est la plus représentative du secteur en érosion (fig. 1).

Dans son ensemble, le système cordon dunaire/plage enregistre des changements morphologiques peu marqués (fig. 4A et 4B). Les variations les plus importantes concernent le haut de plage et le pied de la dune où les modifications atteignent $\pm 45 \mathrm{~cm}$ par rapport au profil moyen (fig. 4C). Le bilan sédimentaire calculé sur l'ensemble du profil pour toute la période d'observation est déficitaire d'environ $16 \mathrm{~m}^{3} / \mathrm{m} .1$ (fig. 4A). Il confirme la tendance enregistrée depuis le début des années 1980 et montre que l'érosion du cordon dunaire est en grande partie liée à un démaigrissement chronique de la plage intertidale qui n'est plus alimentée en sédiment, favorisant ainsi la submersion du pied de dune.

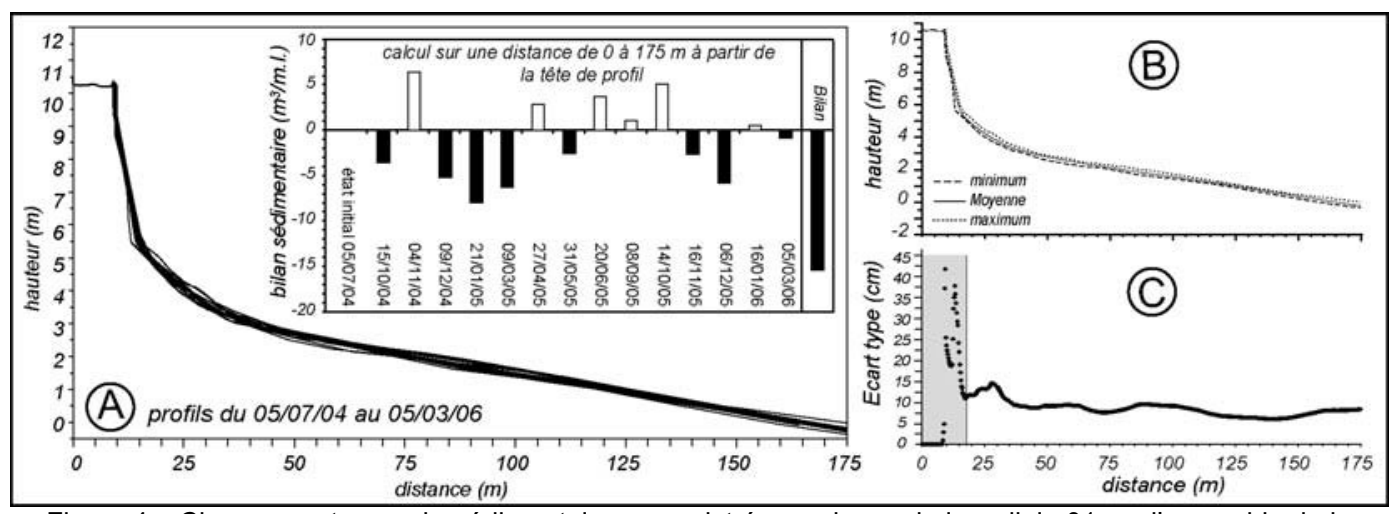

Figure 4 - Changements morphosédimentaires enregistrés au niveau de la radiale 01 sur l'ensemble de la période d'observation $-4 a$ : évolution des bilans sédimentaires $-4 b$ : variations maximales de profils $-4 c$ : importance des variations verticales le long du profil exprimée en écart type.

La même tendance est observée lorsque l'on considère le cordon dunaire stricto sensu. Sur cette partie du profil la déperdition de matériel atteint environ 4 $\mathrm{m}^{3} / \mathrm{m} .1$ sur l'ensemble de la période (fig. 5), ce qui équivaut à un recul total du front de dune de $1 \mathrm{~m} / \mathrm{an}$. Cette valeur confirme par ailleurs les données obtenues par photogrammétrie. Toutefois, dans le détail on observe une forte variabilité saisonnière illustrant ainsi le rôle important des forçages météo-marins dans les 
changements morphosédimentaires. Les hivers 2004-05 et 2005-06 sont marqués par des épisodes de démaigrissement sédimentaire alors que la période intermédiaire, printemps/été 2005, montre un net réengraissement du système dunaire (fig. 5).

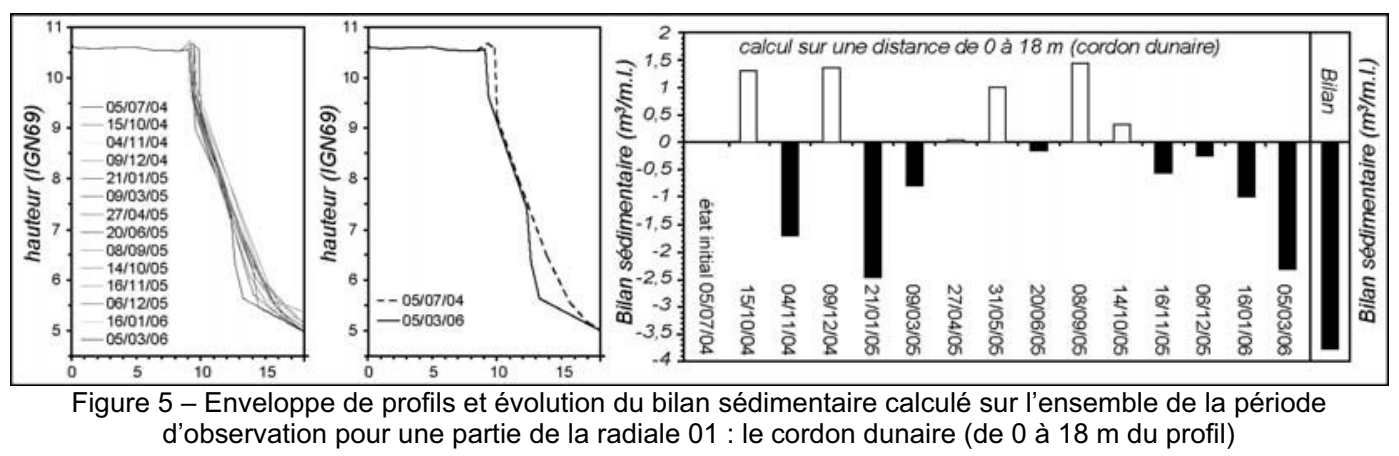

\section{Analyse des niveaux marins extrêmes}

L'analyse des conditions hydrodynamiques et météo-marines enregistrées durant la période d'observation a été réalisée afin de déterminer les niveaux marins extrêmes intervenant dans l'érosion du pied de dune. Cette étude repose sur la combinaison de deux paramètres jouant sur la déformation du plan d'eau : (i) la marée observée (données du marégraphe de Roscoff - fournies par le SHOM-Brest - situé à $40 \mathrm{~km}$ au NE de Guissény) qui enregistre les effets météorologiques (vent et pression barométrique) ${ }^{[5]}$; (ii) l'action de la houle à la côte définie par le run-up, ce dernier étant la somme de deux composantes : les variations du plan d'eau liées à l'agitation (setup : $\bar{\eta}_{\text {max }}$ ) et l'action du jet de rive (wave uprush: $R$ ), soit $R^{T}=\bar{\eta}_{\max }+R^{[4]}$. Le run-up a été calculé à partir de l'équation de P. Ruggiero et al., ${ }^{[6]}$ qui s'applique particulièrement bien au plage à caractère dissipant $(\tan \beta: 0,025)$ :

$$
R^{T}{ }_{2 \%}=0,27 \sqrt{S H_{o} L_{o}}
$$

où $S$ : pente de la plage $(\tan \beta) ; H_{0}$ : hauteur de la houle au large $\left(H_{m o}\right)^{1} ; L_{0}$ : longueur d'onde $=g T^{2} / 2 \pi=1,561 T^{2}\left(g\right.$ accélération de la pesanteur : $9,81 \mathrm{~m} / \mathrm{s}^{2} ; T$ $=T_{p i c}$ : période de la houle en seconde $)^{l}$.

Ces données ont été croisées avec les résultats acquis sur l'évolution du bilan sédimentaire calculé sur la portion du profil de plage correspondant à la dune (de 0 à $18 \mathrm{~m}$ ) (fig. 6). L'altitude du pied de dune ( 5,60 m IGN69) a été prise ici comme niveau de référence. L'analyse consiste à déterminer les épisodes où le niveau marin a été supérieur à ce référentiel. Si l'on considère la déformation du plan d'eau donnée uniquement par la marée observée, on s'aperçoit que le pied de dune n'est jamais atteint par la mer. Si l'on intègre le paramètre run-up, on note

\footnotetext{
${ }^{1}$ Les données de houle ont été produites par simulation numérique à partir du modèle d'états de mer océanique du logiciel TOMAWAC dans le cadre du projet de construction d'un atlas numérique de houle le long des côtes Atlantique Manche Mer du Nord (collaboration entre Météo France, le CETMEF et LNHE-EDF).
} 
que plusieurs épisodes ont dépassé la cote $5,60 \mathrm{~m}$ générant dans tous les cas une érosion du cordon dunaire (fig. 6). Ainsi, l'hiver 2004-2005 est marqué par trois évènements particulièrement morphogènes, les tempêtes du 27 octobre 2004, du 12 janvier 2005 et du 11 février 2005. La longue période de calme qui suit, à partir du mois de mars 2005, a permis à la dune de se régénérer, surtout durant l'été. Il faut attendre les événements du 3 novembre, du 3 et 31 décembre 2005, pour que de nouveau des hauts niveaux marins entraînent l'érosion de la dune. Le niveau maximum obtenu se situe à environ 6,80 m (cote IGN69), soit plus d'un mètre au dessus de la hauteur de pied de dune.

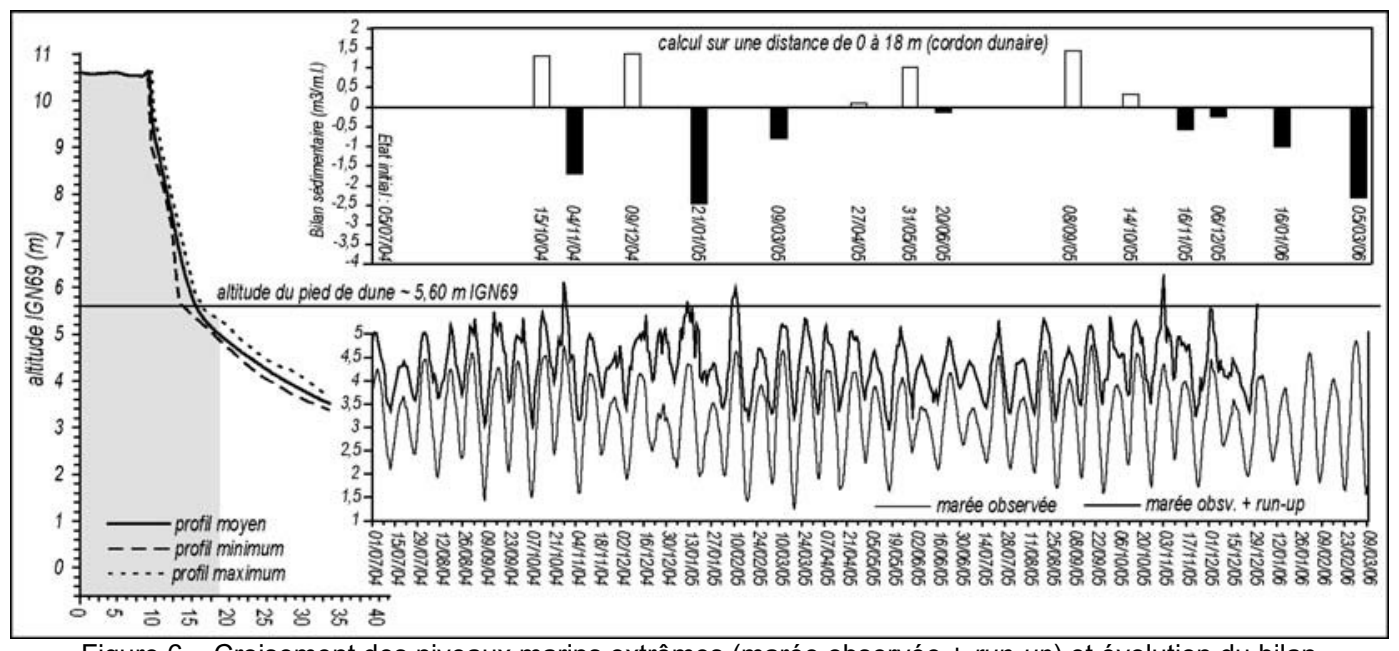

Figure 6 - Croisement des niveaux marins extrêmes (marée observée + run-up) et évolution du bilan sédimentaire de la dune au niveau de la radiale 01 - (les données de vent pour les années 2005 et 2006 n'étant pas disponibles, le calcul du run-up n'a pu être réalisé que pour la période juillet-décembre 2004).

\section{Quelle solution pour lutter contre l'érosion?}

Les solutions techniques retenues tiennent compte des points suivants : (i) le peu d'efficacité d'une technique dure (enrochement) écartant d'emblée toute solution visant à rigidifier le trait de côte ; (ii) l'importance du niveau altimétrique du haut de plage/pied de dune dans les processus d'érosion; (iii) l'existence d'un système globalement déficitaire mais au sein duquel, un fonctionnement saisonnier permet durant l'été le réengraissement du haut d'estran/pied de dune. A partir de là, la solution qui consiste à reconstituer la dynamique sédimentaire et la morphologie du haut d'estran a été préférée. Deux propositions ont été faites et discutées avec l'ensemble des acteurs locaux (élus, riverains, etc.).

La première solution consisterait à exhausser artificiellement l'ensemble du haut d'estran/pied de dune par du rechargement afin de réduire la submersion du pied de dune. Une estimation du volume de sable nécessaire a été réalisée en tenant compte de la hauteur du niveau marin extrême défini précédemment $(6,80$ $\mathrm{m}$ cote IGN69) et de la largeur de plage à recharger. Cette dernière correspond à la partie la plus mobile du haut de plage qui s'étend jusqu'à $55 \mathrm{~m}$ du pied de dune en moyenne. Deux valeurs sont proposées compte tenu des variations saisonnières du profil de plage : 22 à $7,5 \mathrm{~m}^{2}$; ramenées à la distance linéaire concernée de 400 $\mathrm{m}$, on obtient des volumes compris entre 3000 et $9000 \mathrm{~m}^{3}$ (fig. 7). 


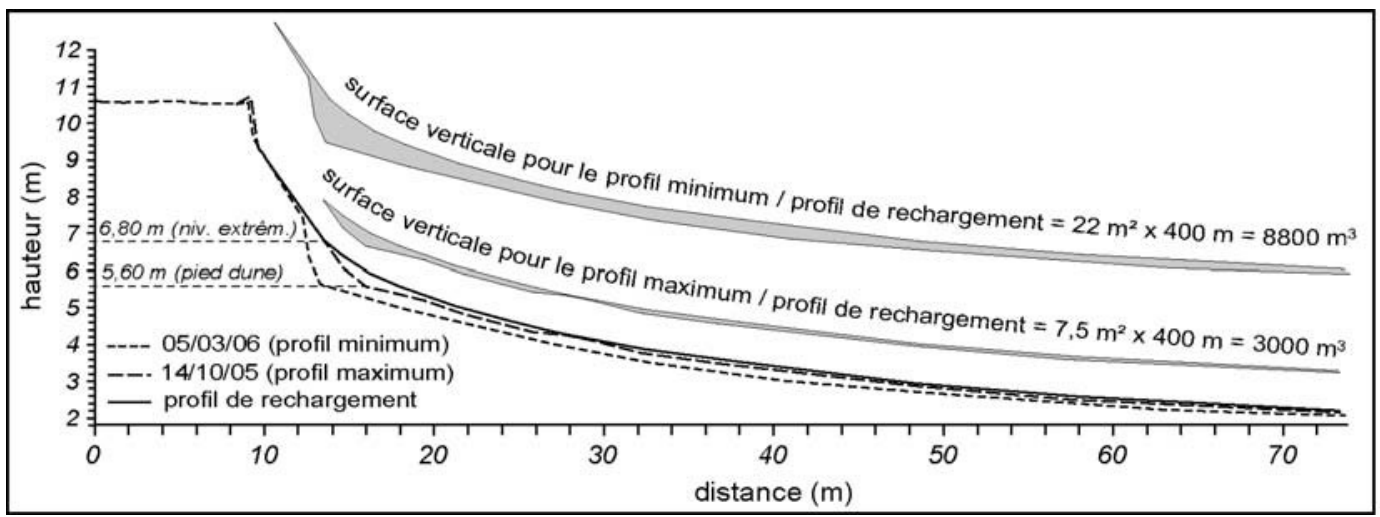

Figure 7 - Estimation des volumes de sable nécessaires à un rechargement de l'ensemble du haut estran / pied de dune ( $1^{\text {ère }}$ solution envisagée)

Cependant, cette première solution ne permettrait pas d'éliminer l'érosion du pied de dune lors d'un évènement particulièrement violent. Pour une stabilisation plus efficace il faudrait recourir à un ouvrage de défense longitudinal de type structure monolithique cylindrique en géocomposite injectée hydrauliquement de granulats (procédé Stabiplage ${ }^{\circledR}$ ou Geotube ${ }^{\circledR}$ ). Cette seconde solution permettrait d'amortir les effets butoir des tempêtes sans pour autant rigidifier le trait de côte. Si l'on tient compte de la hauteur du niveau marin extrême situé à 1,2 $\mathrm{m}$ au dessus du pied de dune, un ouvrage d'un diamètre de 1,5 $\mathrm{m}$ suffirait. La mise en place de ce dernier devrait toutefois s'accompagner d'un rechargement afin de l'enfouir et de reconstituer le profil d'équilibre de la dune (fig. 8). L'estimation des volumes nécessaires a été réalisée suivant la même méthode que celle décrite précédemment, mais sur une largeur plus réduite car seul le versant externe dunaire est concerné $(30 \mathrm{~m}$ à partir de la tête de profil). Les valeurs sont comprises entre 5600 et $8400 \mathrm{~m}^{3}$ (fig. 8).

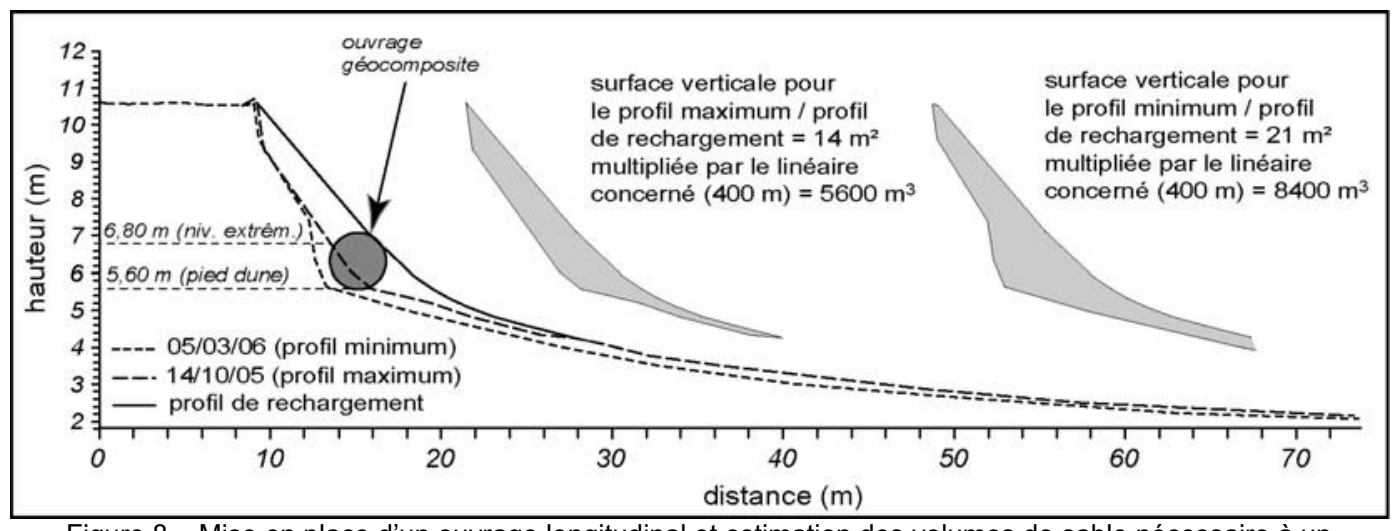

Figure 8 - Mise en place d'un ouvrage longitudinal et estimation des volumes de sable nécessaire à un reprofilage du versant externe de la dune ( $2^{\text {nde }}$ solution envisagée)

\section{Discussion et conclusion}

A travers l'analyse des niveaux marins extrêmes (run-up), cette étude montre combien l'approche fondamentale peut servir la recherche appliquée. Toutefois, une critique peut être faite sur la significativité de ces premiers résultats qui ne portent que sur deux années d'observation. Un approfondissement de la démarche 
mise en œuvre en matière de traitement des données et de proposition de solutions techniques semble nécessaire. D'ors et déjà, une analyse des chroniques météomarines anciennes est envisagée afin de renforcer l'étude des niveaux marins extrêmes. Dans le même temps, la poursuite du suivi jusqu'en 2008 devrait permettre d'améliorer les résultats sur les changements morpho-sédimentaires.

Le choix définitif en matière de solution de défense contre l'érosion dépendra de deux éléments, le coût et la disponibilité d'un stock sédimentaire, en terme de proximité, pour le rechargement. Le coût de mise en place d'une structure en géocomposite en pied de dune, sur 400 mètres de linéaire, peut être évaluée à environ 110.000 euros (enlèvement de l'enrochement existant, pose de la structure en géocomposite, pompage et remplissage en granulat de la structure géotextile). En revanche, l'estimation pour un simple rechargement du haut d'estran/pied de dune est difficile à évaluer car il dépend directement de la disponibilité du stock sédimentaire mobilisable. Les coûts peuvent varier de $30.000 €$ (si le matériel était prélevé à proximité de la zone à recharger), à $600.000 €$ si l'on devait le faire venir par camion.

\section{Bibliographie}

1 Bodéré J.C., Hallégouët B., Piriou N., (1983). Schéma de protection contre la mer du littoral finistérien, Communes de Guisseny et de Plouguerneau, Zone du Curnic, rapport d'étude, Université de Bretagne Occidentale, $81 \mathrm{p}$.

2 Hallégouët B., (1998). Etude du site portuaire du Curnic, propositions pour améliorer le site et réduire les impacts négatifs liés aux ouvrages existants, commune de Guisseny, Finistère, rapport d'étude, Université de Bretagne Occidentale, $21 \mathrm{p}$.

3 Hallégouët B., Yoni C., (1999). La digue du polder du Curnic (Guisseny, Finistère), état des lieux et propositions pour sa restauration, rapport d'étude, Université de Bretagne Occidentale, $53 \mathrm{p}$.

4 Komar P.D., (1998) - Beach processes and sedimentation, $2^{\text {nd }}$ Edit., Printice Hall, 544p.

5 Pirazzoli P.A., (2000) - Surges, atmospheric pressure and wind change and flooding probability on the Atlantic coast of France, Oceanologica Acta, 23(6), 643-661.

6 Ruggiero P., Komar P.D., McDouglas W.G., Marra J.J., Beach R.A., (2001). Wave runup, extreme water levels and erosion of properties backing beaches, Journal of Coastal Research, 17(2), 407-419.

7 Suanez S., Sparfel L., (2005). Rapport d'activité sur le suivi morphosédimentaire du cordon dunaire de la plage du Vougot (commune de Guisseny) pour l'année 2004-2005, GEOMER - LETG UMR 6554 CNRS, Institut Universitaire Européen de la Mer (Plouzané), 24 p. 